\title{
The thermal expansion of hard magnetic materials of the Nd-Fe-B system
}

\author{
Igor Savchenko ${ }^{1, *}$, Yurii Kozlovskii ${ }^{1}$, Dmitriy Samoshkin ${ }^{1}$, and Oleg Yatsuk ${ }^{1}$ \\ ${ }^{1}$ Kutateladze Institute of Thermal Physics, 630090 Novosibirsk, SB RAS, Russia
}

\begin{abstract}
The results of dilatometric measurement of the thermal expansion of hard magnetic materials brands N35M, N35H and N35SH containing as a main component the crystalline phase of $\mathrm{Nd}_{2} \mathrm{Fe}_{14} \mathrm{~B}$ type are presented. The temperature range from 200 to $750 \mathrm{~K}$ has been investigated by the method of dilatometry with an error of $1.5-2 \times 10^{-7} \mathrm{~K}^{-1}$. The approximation dependences of the linear thermal expansion coefficient have been obtained. The character of changes of the thermal coefficient of linear expansion in the region of the Curie point has been specified, its critical indices and critical amplitudes have been defined.
\end{abstract}

\section{Introduction}

A large number of experimental studies are devoted to measuring the thermophysical properties of substances in a magnetic field. It is known that under its influence curious thermal effects are observed in the magnetic materials. In particular there are magnetostrictive and magnetocaloric effects. The aim of the present work was to measure the linear thermal expansion coefficient (LTEC) of some technically important compounds of Nd-Fe-B system, as well as to clarify the question about the influence of the residual magnetization by density and thermal expansion of the samples. The materials which are used to create advanced permanent magnets with record values of the maximum magnetic product were investigated. Literature search revealed that the magnetic properties of iron compounds with neodymium and boron were studied in sufficient detail, which is impossible to tell of their thermal characteristics. Specifically it was succeeded to find only one paper [1], where the measurement results of thermal expansion of amorphous alloy of $\mathrm{Nd}-\mathrm{Fe}-\mathrm{B}$ system are provided; and it was not possible to find data for crystalline phases. The results presented in this paper allow to partially fill the gap.

\section{Experimental technique}

The thermal expansion was investigated on horizontal dilatometer DIL-402C [2] produced by the German company NETZSCH with holder and pushrod made of fused silica. Technique of carrying experiments was described in detail in [3]. The sample mounted on a stand was clamped between the holder and pushrod with a load of $45 \mathrm{cN}$, which was held

* Igor Savchenko: savchenko@itp.nsc.ru, savchenko.igor@gmail.com 
constant during the experiment. The sample length change was registered by an inductive linear variable displacement transducer (LVDT) with a resolution better than $1 \mathrm{~nm}$, and the temperature was measured with Type E thermocouple, which hot junction was located in the vicinity to the lateral surface of the sample. The measurements were carried out in the temperature range $170-780 \mathrm{~K}$ during heating and cooling at a rate of $2 \mathrm{~K} / \mathrm{min}$ and $30 \mathrm{~min}$ isothermal exposure at maximum and minimum temperatures. The temperature and the length change were registered every $2 \mathrm{~s}$, which allowed to obtain the data with a step less than $0.3 \mathrm{~K}$. Before the experiment, the setup is evacuated (up to $1 \mathrm{~Pa}$ ) and filled with helium (99.995 vol. \% pure), which was additionally purged by special device of cleaning and drying of inert gases - EPISHUR-A 11 SL [4]. The nonlinearity of the displacement transducer, differences in the temperatures and in LTECs of the holder and pushrod materials, etc. were taken into account by the measurement of the correction function of the dilatometer. The temperature dependence of the correction function was determined using certified reference samples of fused silica $25 \mathrm{~mm}$ long and $6 \mathrm{~mm}$ diameter certified by NETZSCH company in conditions which were identical to those of the main experiments. The correction function determined in this way was reproduced within $0.2 \mu \mathrm{m}$.

Apparatus, methods of measurement and processing of the results were tested in experiments with high-purity (99.99 mass. \% pure) samples of aluminium and copper. Comparison of the results of measurements with the most credible literature data showed that the deviation in the LTEC values is no more than $(1-1.5) \times 10^{-7} \mathrm{~K}^{-1}$. The control measurements of linear sizes, mass and magnetic field generated by the sample were carried out directly before and after the experiments. The electronic caliper Kraftool [5], electronic indicator Tesa Digico 10 [6], analytical balances AND GH-300 [7] and the magnetometer Aktakom ATE-8702 [8] were used for this measurements.

\section{Investigated materials and sample preparation}

The experiments were performed on samples of brands N35M, N35H and N35SH, this is hard magnetic materials containing as a main component the crystalline phase of $\mathrm{Nd}_{2} \mathrm{Fe}_{14} \mathrm{~B}$ type. The technology of their manufacture is described in [9], and their technical characteristics are listed in [10]. According with the information provided by the manufacturer the samples have the following chemical structure, namely $\mathrm{Fe} \sim 71 \%$, $\mathrm{Nd} \sim 24-27 \%$, Dy $\sim 0.5-2.5 \%$, Co $\sim 1 \%$, B 1\%. According to the composition various brands differ the content of dysprosium, which is $0.5,1$ and $2.5 \%$ for brands N35M, N35H and $\mathrm{N} 35 \mathrm{SH}$ respectively. From the viewpoint of known properties the specimens of different grades distinguish range of the operating temperatures and the value of the coercive force. For our compounds we have that the more of dysprosium in the sample, the higher the coercive force and the upper limit of the permitted temperature interval. The specimens were the shape of cylinders $25 \mathrm{~mm}$ long and $6 \mathrm{~mm}$ diameter with plane base, their protective coating of zinc was mechanically removed before the beginning of the experiments.

\section{Procedure of the experiments and data processing}

Initially for the samples of each brand experiments to determine the temperature dependence of the residual magnetization and finding of maximum working temperature $T_{\max }$ were performed. The maximum working temperature was selected so that irreversible change of residual magnetization was not occurred after heating to this temperature and subsequent cooling down to $293 \mathrm{~K}$. The experiments consisted of successive cycles of heating, isothermal holding and cooling of the initially magnetized sample in the 
dilatometer furnace. The maximum heating temperature was increased for each subsequent cycle, and the magnetic field of the sample was measured in gaps between cycles. Dilatometric experiments with the magnetized samples were carried out at temperatures that did not exceed the maximum working temperature. Thereafter the samples were demagnetized by heating above the Curie temperature $\left(T_{C}\right)$, and measurements of thermal expansion were made in the entire temperature range.

Measurement results are given as the temperature dependence of relative expansion of the sample $\varepsilon$ under heating or cooling:

$$
\varepsilon(T)=\left(L(T)-L_{293}\right) / L_{293},
$$

where $L_{293}$ and $L(T)$ are the sample length at $293.15 \mathrm{~K}$ and at $T$ respectively. The LTEC $\alpha$, and the true LTEC $\alpha^{*}$ are determined as follows:

$$
\begin{aligned}
& \alpha(T)=\frac{1}{L_{293}}\left(\frac{\partial L}{\partial T}\right)_{p}=\left(\frac{\partial \varepsilon}{\partial T}\right)_{p}, \\
& \alpha^{*}(T)=\frac{1}{L}\left(\frac{\partial L}{\partial T}\right)_{p}=\frac{\alpha(T)}{1+\varepsilon(T)},
\end{aligned}
$$

where $p$ is the pressure.

The temperature dependence of the LTEC is found by direct numerical differentiation of the raw experimental data on relative expansion:

$$
\alpha\left(T_{i}\right)=\frac{1}{2}\left(\frac{\varepsilon_{i+1}-\varepsilon_{i}}{T_{i+1}-T_{i}}+\frac{\varepsilon_{i}-\varepsilon_{i-1}}{T_{i}-T_{i-1}}\right),
$$

where $\varepsilon_{i}=\varepsilon\left(T_{i}\right)$ is the relative expansion of the sample at the temperature $T_{i}$. The real interval of determination of the LTEC $\left(T_{i+1}-T_{i}\right)$ is no more than $0.2 \mathrm{~K}$. Such approach allows to get rid errors caused by the ambiguity of the choice depending on the type $\varepsilon(T)$, as well as to identify the features behavior of the linear coefficient of thermal expansion in the field of phase transformations.

In the field of magnetic phase transition a description of the results is carried out using scaling theory [11]. The magnetic contribution is stood out from the values of $\alpha^{*}(T)$ for this purpose:

$$
\alpha_{\mathrm{mag}}^{*}(T)=\alpha^{*}(T)-\alpha_{\mathrm{para}}^{*}(T)
$$

where $\alpha_{\text {para }}^{*}(T)$ is found by the approximation of experimental data in the region of paramagnetic state. The magnetic component of the true LTEC is written in the form:

$$
\alpha_{\text {mag }}^{*}=A|\tau|^{a}+B
$$

where $A, B$ are constants, and $a$ is the critical exponent of the true LTEC, and $\tau=\left(T-T_{C}\right) / T_{C}$ is reduced temperature. It is obvious that $B=\alpha_{\text {mag }}^{*}\left(T_{N}\right)$. Then, introducing a new variable $Y_{\text {mag }}=\alpha_{\mathrm{mag}}^{*}-\alpha_{\mathrm{mag}}^{*}\left(T_{N}\right)$, we obtain from (6): 


$$
\ln \left(Y_{\text {mag }}\right)=\ln (A)+a \ln (|\tau|)
$$

As it is seen from (7), it is possible to calculate the values of the critical amplitude $A$ and the critical exponent $a$ when performing the linear approximation of $\ln \left(Y_{\text {mag }}\right)$ by $\ln (|\tau|)$.

\section{Results and discussion}

The experiments to determine the maximum working temperature showed a good agreement of this parameter with values declared by the manufacturer [10]. According to the results of our measurements the maximum working temperature for brands N35M, $\mathrm{N} 35 \mathrm{H}$ and $\mathrm{N} 35 \mathrm{SH}$ was 373,393 and $423 \mathrm{~K}$ respectively. The results of dilatometric measurements of the sample of brand $\mathrm{N} 35 \mathrm{H}$ in magnetized and demagnetized states are presented in Fig. 1, at temperatures no greater than $T_{\max }$. The measurement results of the relative expansion $\varepsilon(T)$ of demagnetized samples throughout the investigated temperature range are shown in Fig. 2, and the temperature dependences of the LTEC $\alpha(T)$ and the density $\rho(T)$ are illustrated in Fig. 3 and Fig. 4 respectively. As may be seen from the Figure 1, the residual magnetization does not have any influence on the temperature dependence of the relative expansion. The measurement results are practically identical and at the higher temperatures for magnetized and demagnetized samples. A similar pattern is typical for experiments with samples N35M and N35SH brands. The density of the samples under normal conditions are not changed after the demagnetization and takes the following values $\rho_{N 35 M}=7412, \rho_{N 35 H}=7332$ and $\rho_{N 35 S H}=7355 \mathrm{~kg} / \mathrm{m}^{3}$. Thus it can be concluded, that the magnetostrictive effect is negligibly small for test materials at a temperature of $473 \mathrm{~K}$ to $T_{\max }$ and a magnetic field which is not exceeded $400 \mathrm{mT}$. The sharp minimum in the temperature dependence of $\alpha(T)$ is associated with the transition from the ferromagnetic to the paramagnetic state. This phase transition of the second order is clearly seen not only on the LTEC curve, but on the relation of the relative expansion (see Fig. 2).

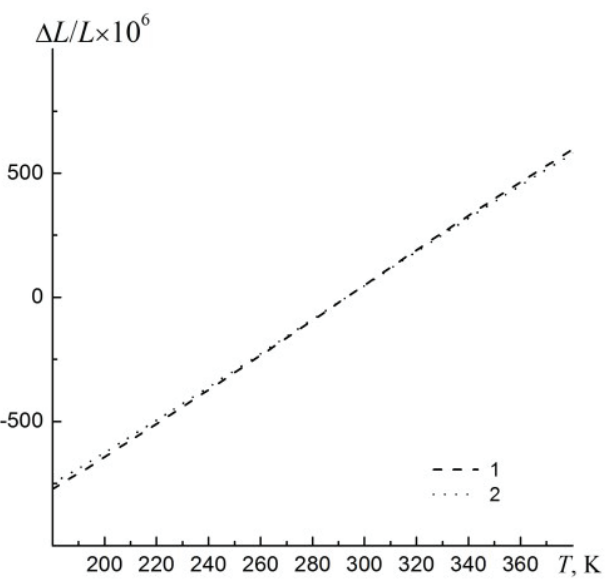

Fig. 1. The results of dilatometric measurements of the sample of brand $\mathrm{N} 35 \mathrm{H}$ in magnetized and demagnetized states, at temperatures no greater than Tmax. 1 - the magnetic field on the surface of the sample is $400 \mathrm{mT} ; 2$ - the magnetic field on the sample surface is $0 \mathrm{mT}$.

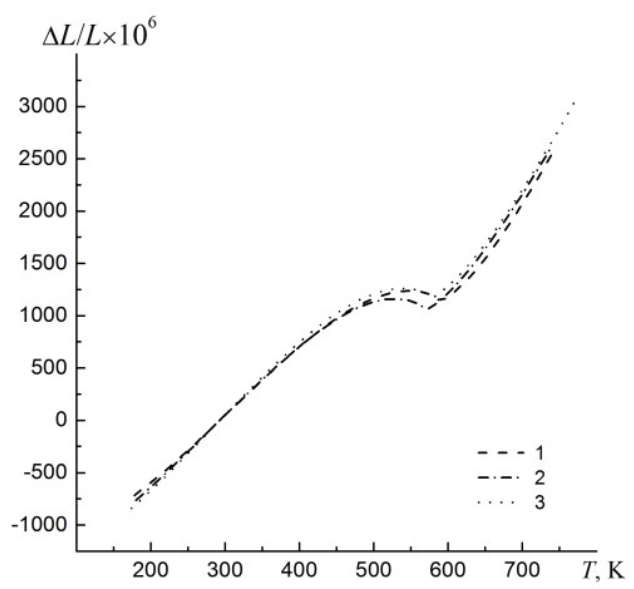

Fig. 2. The measurement results of the relative expansion of investigated materials. $1-\mathrm{N} 35 \mathrm{M}$, 2 - N35H, 3 - N35SH. 


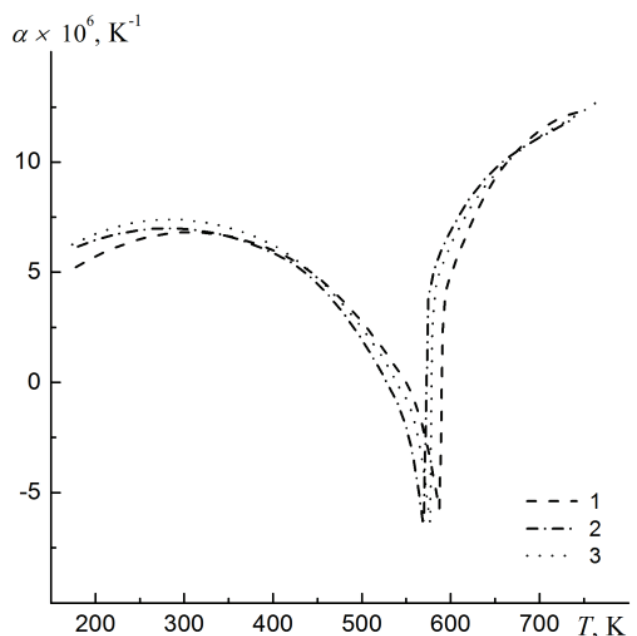

Fig. 3. The linear thermal expansion coefficient. $1-\mathrm{N} 35 \mathrm{M}, 2-\mathrm{N} 35 \mathrm{H}, 3-\mathrm{N} 35 \mathrm{SH}$.

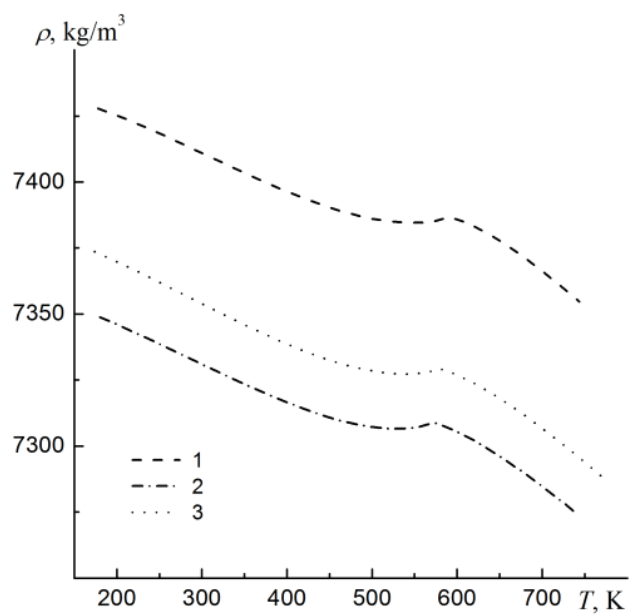

Fig. 4. The density of the samples. $1-\mathrm{N} 35 \mathrm{M}$, $2-\mathrm{N} 35 \mathrm{H}, 3-\mathrm{N} 35 \mathrm{SH}$.

It can be seen from Fig. 3, that it is impossible to describe the dependence of the $\alpha(T)$ on a single relation. Therefore, the entire measurement interval was divided into areas in which the separate data processing was carried out. Outside the temperature intervals directly adjoined to the Curie point the primary data were approximated by the least squares method of the third degree polynomials. The approximations were made using scaling dependencies for the magnetic phase transition area. The variation of the magnitude of $T_{C}$ was performed to clarify the Curie temperature so that in the region adjacent to the Curie point, the experimental data were approximated by the equation (7) with the lowest standard deviation. Finding all the points in the confidence limits of the random error of approximation ( $95 \%$ probability) was also the additional requirement. The values of the phase transition temperature determined in this way amounted to 586.3, 569.4 and $576.4 \mathrm{~K}$ for the brands $\mathrm{N} 35 \mathrm{M}, \mathrm{N} 35 \mathrm{H}$ and $\mathrm{N} 35 \mathrm{SH}$ respectively. Approximations of the LTEC are given in the Table. It was found that the critical indices for ferromagnetic and paramagnetic areas differed significantly, as well as were the same with good accuracy for all of studied materials.

Table. Approximations of the LTEC of hard magnetic materials brands N35M, N35H and N35SH.

\begin{tabular}{|c|c|}
\hline $\begin{array}{l}\text { Temperature } \\
\text { interwal, } \mathrm{K}\end{array}$ & Approximation equation of $\alpha(T), \mathrm{K}^{-1} \times 10^{-6}$ \\
\hline \multicolumn{2}{|r|}{ N35M } \\
\hline $200-525$ & $\alpha(T)=-3.65+0.07 \times T-1.14 \times 10^{-4} \times T^{2}$ \\
\hline $525-586.3$ & $\alpha(T)=-26.6+0.033 \times T+33.3 \times((586.3-T) / 586.3)^{0.5}$ \\
\hline $586.3-625$ & $\alpha(T)=-26.6+0.031 \times T+19 \times((T-586.3) / 586.3)^{0.1}$ \\
\hline $625-750$ & $\alpha(T)=-165.7+0.475 \times T-3.17 \times 10^{-4} \times T^{2}$ \\
\hline \multicolumn{2}{|r|}{$\mathrm{N} 35 \mathrm{H}$} \\
\hline $200-525$ & $\alpha(T)=-2+0.066 \times T-1.17 \times 10^{-4} \times T^{2}$ \\
\hline $525-569.4$ & $\alpha(T)=-22+0.026 \times T+30.6 \times((569.4-T) / 569.4)^{0.5}$ \\
\hline $569.4-625$ & $\alpha(T)=-22+0.026 \times T+18 \times((T-569.4) / 569.4)^{0.1}$ \\
\hline
\end{tabular}




\begin{tabular}{|c|c|}
\hline $\begin{array}{c}\text { Temperature } \\
\text { interwal, } \mathrm{K}\end{array}$ & Approximation equation of $\alpha(T), \mathrm{K}^{-1} \times 10^{-6}$ \\
\hline $625-750$ & $\alpha(T)=-75.1+0.224 \times T-1.44 \times 10^{-4} \times T^{2}$ \\
\hline \multicolumn{2}{|c|}{$\mathrm{N} 35 \mathrm{SH}$} \\
\hline $200-525$ & $\alpha(T)=-1.9+0.066 \times T-1.15 \times 10^{-4} \times T^{2}$ \\
\hline $525-576.4$ & $\alpha(T)=-22.8+0.028 \times T+30.8 \times((576.4-T) / 576.4)^{0.5}$ \\
\hline $576.4-625$ & $\alpha(T)=-22.8+0.026 \times T+18.7 \times((T-576.4) / 576.4)^{0.1}$ \\
\hline $625-750$ & $\alpha(T)=-72.2+0.208 \times T-1.27 \times 10^{-4} \times T^{2}$ \\
\hline
\end{tabular}

\section{Conclusion}

For the first time, new experimental data on the thermal expansion of hard magnetic materials brands $\mathrm{N} 35 \mathrm{M}, \mathrm{N} 35 \mathrm{H}$ and $\mathrm{N} 35 \mathrm{SH}$ containing as a main component the crystalline phase of $\mathrm{Nd}_{2} \mathrm{Fe}_{14} \mathrm{~B}$ type were obtained. The measurements were carried out by dilatometric method with an error of $1.5-2 \times 10^{-7} \mathrm{~K}^{-1}$ at temperature range from 200 to $750 \mathrm{~K}$. The approximation dependences of the linear thermal expansion coefficient were obtained. The character of changes of the thermal coefficient of linear expansion in the region of the Curie point was specified. It was shown that the critical indices for the ferromagnetic and paramagnetic regions vary significantly. The values of critical exponents for all investigated materials coincide. The values of the maximum working temperature of the permanent magnets created from the investigated ferromagnets were identified. It was shown that the presence of the constant magnetic field up to $400 \mathrm{mT}$ did not lead to noticeable changes in the LTEC or the density of investigated materials.

This work supported by the Russian Foundation for Basic Research, project no. 15-38-20223.

\section{References}

1. K. Fukamichi, H. Komatsu, T. Masumoto, T. Kaneko, K. Shirakawa, Y. Satoh, High Pressure Research, 1, 193-201 (1989)

2. http://www.paralab.pt/sites/default/files/pdf/DIL402C.pdf

3. Yu.M. Kozlovskiy, S.V. Stankus, High Temperature, 52, 536-540 (2014)

4. http://granat-e.ru/stend_epishur-a_sl.html

5. http://kraftool-instrument.ru/SHtangencirkul-kraftool-elektronnyj-metallicheskij$150 \mathrm{~mm}-\mathrm{p}-624 . \mathrm{html}$

6. http://technobearing.ru/tesa_digico-10

7. http://a-and-d.ru/product/gh-300

8. http://www.aktakom.ru/kio/index.php?ELEMENT_ID=39662

9. Patent No: US 8182618 B2

10. magnit54.com/biblioteka/harakteristiki_neodimovyh_magnitov

11. F.H. Spedding, B.J. Beaudry, J. Less-Common Metals, 25, 61-73 (1971) 